\title{
Efficacité Technique des Petits Producteurs du Maïs au Bénin
}

\author{
Fawaz A. Adéchinan Aminou, PhD \\ Université d'Abomey-Calavi/ \\ Faculté des Sciences Economiques et de Gestion, Bénin
}

Doi:10.19044/esj.2018.v14n19p109 URL:http://dx.doi.org/10.19044/esj.2018.v14n19p109

\begin{abstract}
This study examines how small maize farmers allocate their production inputs and identifies the elements that characterize efficient farms. A Cobb Douglas stochastic frontier approach is used to estimate farm level technical efficiency of maize farmers. Average technical efficiency of sampled farms was estimated to be $65.40 \%$ with a minimum of $20.47 \%$ and a maximum of $93.46 \%$. The study further showed that the farmers' sex, the use of improved seeds, the maize selling price, the non-farm income, the contact with an NGO, the access to credit and the production area played a positive and significant role in improving technical efficiency. The results imply that government can save resources used in extension services and focus on its improved seed distribution policy. Also, imperfections in the labor/capital market contribute to the low efficiency of farm households.
\end{abstract}

Keywords: Technical efficiency, maize, Benin, stochastic frontier

\section{Résumé}

Cette étude vise à analyser la manière dont les petits producteurs de maïs allouent leurs facteurs de production et à identifier les éléments qui caractérisent les exploitations efficaces. La méthode de frontière stochastique de production Cobb-Douglas est utilisée pour estimer les niveaux d'efficacité technique des producteurs du maïs. Le score moyen d'efficacité technique des producteurs de l'échantillon est estimé à $65,40 \%$ avec un minimum de 20,47\% et un maximum de $93,46 \%$. Les résultats indiquent que le sexe de l'exploitant, l'utilisation des semences améliorées, le prix de vente du maïs, la part du revenu non agricole, le contact avec une $\mathrm{ONG}$, l'accès au crédit et la zone de production jouent un rôle positif et significatif dans l'atteinte de la frontière de production. Nos résultats nous amènent à suggérer au gouvernement d'économiser les ressources utilisées dans le cadre des services de vulgarisation et se focaliser sur sa politique de distribution des semences 
améliorées. Egalement, les imperfections sur le marché du travail et du capital contribuent à la faible efficacité des ménages agricoles.

Mots clés: Efficacité technique, maïs, Bénin, frontière stochastique

\section{Introduction:}

Dans la théorie microéconomique, l'objectif des firmes est d'atteindre un output maximal pour une quantité donnée d'inputs avec un coût minimum. Cette notion microéconomique stipule ou suppose que les firmes dans le cadre des règles du libre marché devraient allouer les inputs et l'output de manière efficiente dans le but d'obtenir un profit maximum et/ou un coût minimum (Erkoc, 2012). La mesure de l'efficacité technique fournit des informations utiles sur la compétitivité des exploitations et le potentiel d'amélioration de la productivité, avec les ressources et le niveau de technologie existants (Otieno et al. 2012).

Très peu diversifiée, l'économie béninoise dépend trop largement du secteur agricole qui occupe 36\% du Produit Intérieur Brut (PIB) et 88\% des recettes d'exportation et $15 \%$ des recettes de l'Etat [Ministère de l'Agriculture, de l'Elevage et de la Pêche (MAEP), 2018] ${ }^{7}$. Mais ce secteur tarde à se moderniser et se diversifier. En effet, le secteur agricole béninois est dominé par la filière coton qui occupe entre 25 et $40 \%$ des exportations totales et $34,7 \%$ des recettes d'exportation officielles (BM, 2010). Mais ses performances sont minées par des problèmes d'organisation, les aléas climatiques et l'archaïsme des outils de production. La crise de la filière coton véritablement observable depuis la campagne 1999-2000 a mis à nu la fragilité de l'économie béninoise basée sur un seul produit d'exportation (PPAB ${ }^{8}$, 2001). Cette fragilité a été renforcée par les différentes crises alimentaires et économiques subies par les pays en développement et surtout la crise alimentaire de 2006-2008.

Dès lors, la diversification agricole et la production vivrière sont devenues une priorité pour les acteurs du développement du secteur agricole. C'est en ce sens que le Bénin est entrain de promouvoir d'autres filières porteuses dont le maïs. En effet, le maïs est à ce jour le premier produit alimentaire au Bénin loin devant le riz et le Sorgho (EMICoV $\left.{ }^{9}, 2011\right)$. Ce qui montre l'importance de cette céréale pour la sécurité alimentaire. Compte tenu de son importance pour la sécurité alimentaire et l'économie nationale, le gouvernement lui accorde une place importante dans son document de stratégie de croissance et de réduction de la pauvreté. Dans ce document, le gouvernement s'est clairement fixé comme objectif, pour 1'horizon 2011, d'accroitre la production

${ }^{7}$ www.agriculture.gouv.bj consulté le 17/06/18 à $15 \mathrm{~h} 50$

${ }^{8}$ Programme de Professionnalisation Agricole du Bénin

${ }^{9}$ Enquête Modulaire Intégrée sur les Conditions de Vie des ménages 
de maïs pour passer de 841000 tonnes en 2005 à 1100000 tonnes de manière à atteindre un solde vivrier d'au moins 250000 tonnes. Le maïs est également retenu comme filière prioritaire de relance du secteur agricole où il est envisagé à l'horizon 2015 que le Bénin produise en moyenne 1900000 tonnes de maïs par an et participe durablement aux échanges commerciaux de céréales dans les pays de la sous-région et d'ailleurs (SCRP $\left.{ }^{10}, 2007\right)$.

Ainsi, des programmes d'accroissement de la productivité et de la production agricole ont été mises en place à travers la distribution des engrais, la mise à disposition des producteurs des semences et l'attribution des parcelles aux producteurs. Mais ces différentes politiques ont eu une portée limitée puisqu'à l'échéance du terme (2011-2015), l'objectif du Bénin concernant le maïs n'a pas été atteint car la production du maïs est de 1438918 $\mathrm{kg}$ en 2015 (ONASA $^{11}$, 2016) et toutes les potentialités économiques de la filière ne sont malheureusement pas encore bien valorisées. En effet, comme la production, le rendement du maïs a connu une amélioration passant de 600 $\mathrm{kg} /$ ha en moyenne en 1970 à $1400 \mathrm{~kg} /$ ha en $2009\left(\mathrm{ONS}^{12}, 2010\right)$ puis à 1103 $\mathrm{kg} / \mathrm{ha}$ en $2010 ; 1422 \mathrm{~kg} / \mathrm{ha}$ en $2011 ; 1251 \mathrm{~kg} / \mathrm{ha}$ en 2012 et $1346 \mathrm{~kg} / \mathrm{ha}$ en 2013 (FAOSTAT, 2015). Ce rendement des plantations est toujours faible comparativement aux autres régions du monde comme le Burkina Faso où le rendement du maïs est de $1434 \mathrm{~kg} /$ ha en $2010 ; 1536 \mathrm{~kg} / \mathrm{ha}$ en $2011 ; 1839$ $\mathrm{kg} / \mathrm{ha}$ en 2012 puis de $1799 \mathrm{~kg} / \mathrm{ha}$ en 2013 (FAOSTAT, 2015) et que les producteurs ont une faible maitrise des coûts de production. En dépit de cette amélioration du rendement du maïs, force est de constater que ce rendement connaît une évolution en dents de scie qui fait fluctuer son solde vivrier dans des proportions parfois inquiétantes. Ce qui fait peser des menaces tant sur la sécurité alimentaire que sur les revenus des producteurs et par ricochet sur leur niveau de pauvreté puisque pour réduire la pauvreté au Bénin, il faudrait une augmentation des revenus des $70 \%$ d'actifs employés en agriculture $\left(\mathrm{PEA}^{13}\right.$, 2012). Ceci en raison du fait que chaque augmentation de la productivité agricole de $1 \%$ en Afrique, réduit la pauvreté de $0,6 \%$ et l'augmentation de la production de $1 \%$ fait baisser le nombre de personnes vivant avec moins d'un dollar par jour de 6 millions (Thirtle et al. 2003).

Ces résultats mitigés de ces différents programmes pourraient s'expliquer par le fait que ce sont des programmes qui s'appliquent de façon générale sur l'étendue du territoire national sans tenir compte des spécificités liées à chaque région en termes de contraintes et conditions de production auxquelles font face les producteurs. Aussi, les combinaisons des facteurs de production et des associations des cultures sous optimales pourraient en être une des raisons. De

\footnotetext{
${ }^{10}$ Stratégie de Croissance et de la Réduction de la Pauvreté

${ }^{11}$ Office National de Sécurité Alimentaire

${ }^{12}$ Office National de Soutien des revenus agricoles

${ }^{13}$ Perspectives Economiques d'Afrique
} 
plus, le maïs se cultive toujours dans un contexte de dégradation constante des terres, caractérisé par la persistance des méthodes traditionnelles et une méconnaissance d'organisation d'équipements améliorés de transformation couplée avec le faible niveau des acteurs (ONS, 2010). Cette situation se traduit par une faible valorisation du potentiel économique de cette filière face à des demandes non satisfaites pour l'aviculture, la brasserie, la fabrication des farines infantiles, et provenant des pays limitrophes (Niger, Nigéria...).

De plus, de nombreux pays du continent y compris le Bénin sont importateurs de produits alimentaires dont le maïs avec un niveau d'importation de 1058,3 tonnes et 1272,55 tonnes respectivement en 2013 et 2014 (INSAE, 2016). Ainsi, se concentrer efficacement sur la production de maïs a le potentiel de traiter la question non seulement de l'insécurité alimentaire mais aussi celle du volume déraisonnablement élevé des importations de produits alimentaires. Il importe donc de savoir si les différentes unités de production du maïs du Bénin sont efficaces dans l'utilisation des ressources disponibles. En effet, l'augmentation du volume de la production à travers une augmentation des ressources productives (superficies emblavées en occurrence) n'est pas une option durable. L'augmentation de la production n'exige pas forcement une augmentation globale des ressources productives mais peut aussi résulter d'une modification de la façon de gérer les ressources existantes. Cette étude permettra d'identifier les éléments pouvant améliorer la production, de formuler, compte tenu des caractéristiques de chaque région, des recommandations aussi bien qu'à l'endroit des producteurs qu'aux autorités chargées de mettre en place des politiques agricoles.

\section{Objectifs et hypothèses de recherche:}

Cette étude se propose d'analyser l'efficacité technique des petits producteurs du maïs au Bénin en adoptant une méthode d'estimation de seconde étape qui diffère de l'approche standard la plus utilisée. De façon spécifique, l'étude vise à : (1) déterminer le niveau d'efficacité technique des petits producteurs du maïs (2) décomposer les niveaux d'efficacité technique des producteurs suivant les zones agro-écologiques, la variété de semences utilisées (3) identifier les variables socio-économiques et techniques qui caractérisent les exploitations efficaces. Le papier vise à tester les hypothèses suivantes : (i) les producteurs du maïs peuvent élever leur niveau d'efficacité en changeant leur combinaison des facteurs de production; (ii) le degré d'efficacité diffère selon la zone agro-écologique et selon les semences utilisées et (iii) les variables socio-économiques et techniques telles que l'accès au crédit, l'âge, l'alphabétisation, le contact avec les agents de vulgarisation, utilisation des semences améliorées, le prix de vente du maïs ainsi que le climat déterminent les producteurs efficaces. 


\section{Revue de littérature:}

Les études d'efficacité des exploitations agricoles ont une longue histoire, et ont été basées sur des méthodes de plus en plus évoluées et les avancées méthodologiques en DEA et SFA. Nous présentons dans cette partie, le cadre théorique sur la notion d'efficacité et les travaux empiriques sur l'efficacité des exploitations agricoles.

\section{Cadre théorique sur la notion d'efficacité}

L'efficacité recouvre des éléments larges et parfois différents. Il intègre un ensemble de notions plus précises de production, de coût ou de prix, de profit etc. La frontière reflète la quantité maximale d'output pouvant être atteinte pour un niveau donné d'inputs (frontière de production); le coût minimal de production de l'output pour un niveau donné de prix des inputs (frontière de coût) ; ou le profit maximal pouvant être atteint pour un niveau donné des prix d'output et d'inputs (frontière de profit). Dans tous les cas, la technologie et les facteurs fixes sont aussi considérés comme donnés. C'est avec Leibenstein (1966) à travers la notion de " $X$-efficiency » que le comportement optimal non systématique des producteurs a été considéré. En termes d'analyse comparative, la frontière incarne les meilleures pratiques.

La littérature économique identifie deux types d'efficacités à savoir l'efficacité économique et l'efficacité d'échelle. L'efficacité économique est composée de l'efficacité technique et de l'efficacité allocative. Selon Koopmans (1951), un producteur est techniquement efficace si l'augmentation d'un quelconque output nécessite la diminution d'au moins un autre output ou l'accroissement d'au moins un input, et si une baisse de n'importe quel input exige la hausse d'au moins un autre input ou la réduction d'au moins un output. En d'autres termes, une firme techniquement efficace doit se situer à la frontière de son ensemble de production.

De façon plus précise, c'est Farrell (1957) qui définit l'efficacité en dissociant ce qui relève de la technique de ce qui relève d'un mauvais choix par rapport au prix des intrants. L'efficacité technique mesure ainsi la manière dont la firme combine les facteurs de production lorsque leurs proportions d'utilisation sont données. Il y a inefficacité technique lorsqu'on pourrait obtenir le même niveau d'output avec moins d'intrants. L'efficacité-prix mesure la manière dont le producteur fixe les proportions des différents intrants entrant dans la combinaison productive en se basant sur leurs prix respectifs. Cette mesure donne une appréciation de la façon dont les firmes allouent leurs ressources productives par rapport à un objectif de production. D'où l'appellation d'efficacité allocative souvent utilisée en lieu et place du terme d'efficacité-prix employé par Farrell. En d'autres termes, l'efficacité allocative peut être définie comme l'aptitude des producteurs à choisir les inputs dans les proportions optimales. 
Une décomposition de l'efficacité économique en une composante technique et une composante allocative a été proposée par Farrell (1957) à travers une représentation pédagogique. L'inefficacité technique correspond à l'utilisation d'une quantité d'input supérieure au nécessaire pour un niveau d'output donné. Elle est évaluée par l'écart à la frontière formée par les firmes les plus performantes de l'échantillon. L'inefficacité allocative mesure l'utilisation des inputs dans les proportions qui ne correspondent pas à l'optimalité décrite par les prix relatifs des inputs.

L'obtention simultanée des efficacités technique et allocative est une condition nécessaire et suffisante pour parler d'efficacité économique. Il est possible pour une firme d'être efficace techniquement ou allocativement sans l'être économiquement. L'efficacité économique apparaît donc comme la résultante entre l'efficacité technique et l'efficacité allocative, composantes exclusives et exhaustives de l'efficacité économique (Adegbola et al. 2005). L'efficacité d'échelle quant à elle détermine dans quelle mesure une unité de production fonctionne avec des rendements d'échelle croissants ou décroissants, ce qui permet de définir la taille optimale d'une unité de production.

La théorie économique offre de nombreuses procédures pour l'évaluation de l'efficacité. Deux techniques sont plus couramment utilisées pour estimer la frontière. Nous avons la méthode Data Envelopment Analysis (DEA) et la méthode Stochastic Frontier Analysis (SFA).

La méthode SFA est une méthode de modélisation économique. Elle tient son point de départ des modèles de la frontière de production stochastique introduits simultanément par Aigner et al. (1977) et Meeusen et Van den Broeck (1977). Les principales différences entre les approches DEA et SFA se trouvent exactement dans les deux caractéristiques de la méthode DEA indiquées ci-dessus. Tandis que la méthode DEA est déterministe et non paramétrique, la méthode SFA suppose une relation stochastique entre l'input et l'output et elle est une approche paramétrique. Le principal avantage de la méthode SFA est qu'elle n'attribue pas l'écart de la frontière estimée complètement à l'inefficacité. La distance entre la combinaison d'input/d'output observée peut être attribuée en partie à l'inefficacité et en partie à l'erreur dans les données, résultant par exemple d'une mesure imprécise des inputs et/ou outputs. Cette caractéristique se fait au prix de la nécessité d'imposer une forme fonctionnelle pour permettre l'estimation des paramètres du modèle. Selon Coelli et al. (1998) 1'approche SFA est plus appropriée dans les applications agricoles, en particulier dans les pays en développement, étant donné que l'agriculture dépend fortement des phénomènes aléatoires (le climat). Un problème important avec la SFA lorsqu'on l'applique à des données agricoles de pays en développement est qu'elle n'admet pas d'intrants nuls. Cette approche sera adoptée dans cette 
étude car elle est souvent préférable pour analyser l'efficacité dans l'agriculture puisque les facteurs aléatoires non observés agissent sur la production agricole, et les données au niveau de l'exploitation agricole contiennent généralement des erreurs de mesure considérables (Nasim et al. 2014). De plus, nos données nous permettent de le faire.

\section{Travaux empiriques sur l'efficacité des exploitations agricoles}

Très peu d'études d'efficacité dans l'agriculture utilisent la méthode DEA (par exemple Dhungana et al. 2004 ; Djimasra, 2010 ; Ogundari, 2013). La plus importante littérature porte sur la méthode SFA. Les études de Alene et Hassan (2006) ; Dinar et al. (2007); de Magalhães et al. (2011); Chen et al. (2009); Idiong (2007); Abdulai et Tietje (2007) ; Liu et Myers (2009) et Nasim et al. (2014) utilisent cette méthode en Agriculture.

Utilisant la fonction de frontière stochastique dans une étude d'efficacité technique des inputs spécifiques à chaque district de l'Inde, Venkataramani et $a l$. , (2006) en utilisant une fonction de production de type Cobb-Douglas ont trouvé qu'une amélioration de la santé est associée à une augmentation significative de l'efficacité technique. Des résultats similaires sont obtenus par Loureiro (2009) qui a trouvé que les différences en santé des agriculteurs expliquent la variance dans l'efficacité de la production agricole en Norvège.

Par ailleurs, dans une étude comparative entre les exploitations pauvres et non pauvres, Ahmad (2003) a montré que l'élasticité de production des terres est sensiblement plus élevée dans les fermes riches par rapport aux fermes appartenant à des agriculteurs pauvres. En outre, le coût moyen de l'existence d'inefficacités techniques était d'environ $43 \%$ en termes de pertes de production, avec de grandes variations entre les fermes allant de 17 à $62 \%$. Il a conclu aussi que le groupe le moins performant ne fonctionnait pas uniquement en deçà de la frontière mais également à la partie inférieure de la frontière de production. Par conséquent, l'augmentation de l'accès aux intrants serait susceptible d'augmenter la productivité et réduire la pauvreté. De plus, Costa et al., (2013) ont étudié la relation entre la productivité agricole et la sécurité alimentaire des ménages dans les régions métropolitaines brésiliennes, en tenant compte des autres facteurs individuels. Ils ont constaté que les gains de productivité étaient associés à une plus grande sécurité alimentaire des ménages, dans des proportions faibles dues à la forte influence des caractéristiques particulières telles que l'éducation et le revenu.

Dans l'optique d'identifier les déterminants de l'efficacité technique, Koirala et al. (2013) ont utilisé la forme fonctionnelle Cobb-Douglas pour les producteurs du riz aux Philippines. Les résultats ont montré que le carburant, les engrais, la rente foncière, la saison de plantation et la superficie des terres sont les facteurs qui influent sur la production et l'efficacité technique de la production du riz. Ils ont trouvé un score moyen d'efficacité technique de 0.54. 
Une étude similaire a été faite par Mohammed et Saghaian (2014) sur la production du riz en Corée du Sud. Il ressort de leur étude qu'il était possible d'augmenter l'efficacité de production et que l'effet de l'emplacement était significatif sur le rendement de la production.

Au Bénin, Toléba et al. (2016) ont estimé l'efficacité technique d'un échantillon de 411 exploitations maïzicoles dans les principales zones de production au Bénin en utilisant le modèle de la frontière stochastique de production de type Cobb-Douglas. Les résultats indiquent, d'une part que le score d'efficacité technique varie de 37,37 à $96,22 \%$ avec un score moyen de $80,35 \%$ et d'autre part, que les variables telles l'accès aux engrais et aux herbicides, l'utilisation de la traction animale et du tracteur, l'encadrement technique et l'accès au crédit influencent l'efficacité technique des exploitations de l'échantillon. La présente recherche s'inscrit dans la continuité de celle de Toléba et al. (2016) en se focalisant sur les petites exploitations qui constituent une grande partie des producteurs béninois. De plus, elle propose une analyse suivant les zones agro écologiques et le type de semences utilisées. Mieux, les études citées ci-dessus y compris celle de Toléba et al. (2016) utilisent le modèle Tobit, dans une deuxième étape, pour identifier les déterminants de l'inefficacité. Ceci est économétriquement biaisé car le modèle Tobit suppose une double censure au niveau des scores d'efficacité. Ce qui n'est pas le cas puisque les scores ne sont rien que des proportions qui sont naturellement comprises entre 0 et 1 (Baum, 2008 ; Ramalho et al. 2010) ${ }^{14}$. Ainsi, dans notre étude, le modèle de régression fractionnée (Fractional Regression Model) développé par Papke et Wooldridge (1996) serait utilisé pour l'estimation de deuxième étape de la méthode SFA.

\section{Méthodologie et données:}

Afin d'estimer l'efficacité technique, une fonction de production est utilisée pour que l'efficacité soit analysée ${ }^{15}$. Une utilisation non optimale des facteurs de production qui peut être mise en avant pour les agriculteurs béninois (fortes contraintes sur les marchés du travail et de crédit) implique une inefficacité technique bien connue comme l'inefficience-X (Leibenstein, 1966). En considérant que le producteur $i$ utilise plusieurs intrants $X$ pour produire un seul ou plusieurs outputs $Y$, une fonction de production peut être écrite pour représenter une technologie particulière: $Y_{i}=f\left(x_{i}\right)$ où $f\left(x_{i}\right)$ est une

\footnotetext{
${ }^{14}$ Nous y reviendrons plus en détail au niveau de la méthodologie.

${ }^{15} \mathrm{La}$ productivité agricole peut généralement être décomposée en deux éléments: un dynamique et l'autre statique. Le premier élément est lié au progrès technique et le second à l'efficacité productive. Pour analyser le premier élément, il est nécessaire de disposer de séries chronologiques. Nos données ne nous permettent pas d'avoir une dimension temporelle ainsi seule l'efficacité productive de l'exploitation peut être analysée.
} 
frontière de production. Sur la frontière, le producteur produit l'output maximal pour un ensemble d'inputs donné ou utilise un niveau minimum d'inputs pour produire un niveau donné d'output. En théorie microéconomique, une absence d'inefficacité dans l'économie signifie que toutes les fonctions de production sont optimales et que toutes les firmes produisent à la frontière. Mais si les marchés sont imparfaits, les producteurs peuvent se retrouver en dessous de la frontière de production.

Une mesure orientée output de l'efficacité technique donne l'efficacité technique d'un agriculteur $i$ comme suit:

$$
T E_{i}(x, y)=\left[\max \phi: \phi y \leq f\left(x_{i}\right)\right]^{-1}
$$

Le paramètre $\phi$ est l'expansion de l'output maximal avec l'ensemble des inputs $x_{i}$.

Suivant Kumbhakar et Lovell (2000), l'équation 1, appliquée dans un modèle économétrique donne :

$$
Y_{i}=f\left(X_{i}, \beta\right) \cdot e^{-W_{i}}
$$

Où $Y_{i}$ est un scalaire d'output, $X_{i}$ un vecteur des intrants utilisés par le producteur $i=1, \ldots \ldots \ldots, N$ et $f\left(X_{i}, \beta\right)$ est la frontière de production ${ }^{16}$ où $\beta$ est un vecteur de paramètres technologiques à estimer. $W_{i}$ est une variable aléatoire non observable et non-négative associée à l'inefficacité technique de production qui suit une distribution arbitraire ${ }^{17}$.

Selon Aigner et al. (1976), Meeusen et Van den Broeck (1977), une frontière de production stochastique est utilisée de sorte que le terme d'erreur ait deux composantes: les chocs aléatoires $V_{i}$ (non attribués à la relation entre les inputs et l'output) et l'inefficacité $U_{i}{ }^{18}$. Par conséquent, l'équation 2 devient:

$$
Y_{i}=f\left(X_{i}, \beta\right) \cdot e^{-U_{i}} \cdot e^{V_{i}}
$$

Où $V_{i}$ représente les chocs aléatoires qui sont supposés être indépendants et identiquement distribués avec une distribution normale de moyenne nulle et de variance inconnue. Sous cette hypothèse, un producteur en dessous de la frontière n'est pas totalement inefficace car les inefficacités peuvent aussi être le résultat des chocs aléatoires (comme les chocs climatiques).

Puisque $T E_{i}$ est une mesure orientée output de l'efficacité technique, une mesure de $T E_{i}$ est donnée par :

\footnotetext{
${ }^{16}$ La frontière de production a les propriétés traditionnelles de la monotonicité, la continuité et la concavité (Fuss et McFadden, 1978).

${ }^{17}$ Nous choisissons la distribution exponentielle.

${ }^{18}$ Une frontière déterministe implique un seul terme d'erreur qui est l'inefficacité. L'écart à la frontière est uniquement dû à l'inefficacité.
} 


$$
T E_{i}=\frac{\operatorname{Pr} o d_{o b s}}{\operatorname{Pr} o d_{\text {max }}}=\frac{f\left(X_{i}, \beta\right) \cdot e^{-U_{i}} \cdot e^{V_{i}}}{f\left(X_{i}, \beta\right) \cdot e^{V_{i}}}=e^{-U_{i}}
$$

L'efficacité technique est donc estimée en utilisant le modèle de frontière stochastique donné par les équations 3 et 4 .

L'objectif de la réalisation du modèle de frontière stochastique n'est pas seulement de déterminer les scores d'efficacité technique, mais aussi d'étudier les facteurs qui caractérisent des différences d'efficacité. En s'inspirant de Kumbhakar et al. (1991), Huang et Liu (1994), et Battese et Coelli, (1995), l'équation de l'inefficacité du producteur en relation avec ces facteurs peut être formulée comme suit :

$$
U_{i}=Z_{i} \beta+\varepsilon_{i}
$$

Où $Z_{i}$ est un vecteur des variables sociodémographiques incluant l'âge, la taille du ménage, l'appartenance à un groupement, l'utilisation des semences améliorées, le prix de vente du maïs, l'accès au marché, le revenu non agricole, l'accès aux services de vulgarisation, contact avec une ONG, l'accès au crédit (formel ou informel), le statut d'alphabétisation et la zone de production. $\beta$ est le vecteur des paramètres à estimer et $\varepsilon_{i}$ est le terme d'erreur qui suit une distribution normale tronquée défini par $\varepsilon_{i} \leq-Z_{i} \beta$.

L'estimation du vecteur de paramètres $\beta$ a fait l'objet de débats dans les études d'efficacité. La procédure la plus utilisée consiste dans un premier temps à estimer les indices d'efficacité et dans un second temps procéder à leur régression contre les différents facteurs soupçonnés. Toutefois, lorsque Ray (1988) et Kalirajan (1991) défendent cette procédure à deux étapes, Kumbhakar et al. (1991) ; Battese et al. (1996) la critiquent en argumentant qu'elle viole l'une des hypothèses de base, selon laquelle " les effets d'inefficience sont identiquement et indépendamment distribués de la frontière stochastique ». Battese et Coelli (1995) ont proposé une modélisation à une étape dans laquelle les effets d'inefficience sont fonction de diverses variables observables telles que l'âge, l'éducation, l'accès aux services de vulgarisation, le type de semences utilisées etc. Malgré ces critiques, la procédure à deux étapes est restée populaire dans la recherche des facteurs affectant les indices d'efficacité. Cette popularité est confortée par le fait qu'on n'a pas une liste exhaustive des déterminants de l'inefficacité.

Afin d'estimer l'efficacité technique des producteurs, la présente étude adopte la procédure en deux étapes. Le choix du modèle de régression pour la deuxième étape de l'analyse n'est pas trivial. L'approche standard consistant à utiliser un modèle Tobit à double censure (en 0 et 1 ) pour modéliser les scores est discutable. En effet, l'accumulation d'observations à l'unité est une conséquence naturelle de la façon dont les scores d'efficacité sont définis plutôt que le résultat de la censure. Aussi, le domaine du modèle Tobit à 
double censure diffère-t-il de celui des scores car généralement les scores d'efficacité 0 ne sont pas observés. Cette différence est particulièrement pertinente parce que l'application du modèle Tobit à double censure dans ce contexte revient en fait à l'estimation d'un Tobit à une censure du score

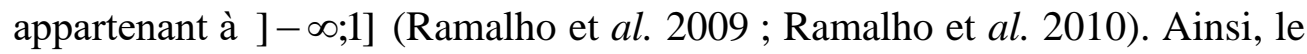
modèle de régression fractionnée (Fractional Regression Model) développé par Papke et Wooldridge (1996) est utilisé pour l'estimation de deuxième étape puisque les scores d'efficacité constituent des proportions ${ }^{19}$.

La méthode de frontière stochastique requiert une spécification préalable d'une fonction la plus généralement utilisée comme Cobb-Douglas et Translog. La fonction Cobb-Douglas est une forme particulière de la fonction de production Translog où les coefficients des termes au carré et d'interaction des variables d'inputs de la fonction Translog sont supposés être égaux à zéro. La frontière Translog est susceptible de multicollinéarité bien qu'elle soit la forme la plus flexible (Thiam et al. 2001). La fonction de production CobbDouglas, en dépit de ses propriétés restrictives, est préférée parce que ses coefficients représentent directement l'effet d'une variation de la quantité des inputs sur l'output et sont faciles à interpréter et à estimer que la frontière Translog (Coelli et Battese, 1998). Ainsi, dans cette recherche, la frontière Cobb-Douglas est préférée.

La fonction de production stochastique Cobb-Douglas traditionnelle utilisée pour estimer l'efficacité technique a la forme générale suivante ${ }^{20}$ (Christensen et al. 1971):

$$
\ln \left(Y_{i}\right)=\beta_{0}+\sum_{j} \beta_{j} \ln \left(X_{i j}\right)-U_{i}+V_{i}
$$

Où $i=1,2, \ldots \ldots, N$ est l'observation de l'unité de l'agriculteur; $j ; k=1, \ldots \ldots ., 9$ sont les inputs utilisés; $\ln \left(Y_{i}\right)$ est le logarithme de l'output de l'agriculteur $i$ et $\ln \left(X_{i j}\right)$ est le logarithme de l'input $j$ utilisé par le $i^{\text {ième }}$ agriculteur et $\beta_{0} ; \beta_{j}$ sont des paramètres à estimés. En outre, la frontière de production nécessite la monotonicité et la concavité. Ces hypothèses doivent être vérifiées à posteriori en utilisant les paramètres estimés pour chaque point de données.

Le modèle empirique final estimé dans ce cas est :

$$
\begin{gathered}
\ln \left(Y_{i}\right)=\beta_{0}+\beta_{1} \ln \left(X_{i 1}\right)+\beta_{2} \ln \left(X_{i 2}\right)+\beta_{3} \ln \left(X_{i 3}\right)+\beta_{4} \ln \left(X_{i 4}\right)+\beta_{5} \ln \left(X_{i 5}\right)+\beta_{6} \ln \left(X_{i 6}\right) \\
+\beta_{7} \ln \left(X_{i 7}\right)+\beta_{8} \ln \left(X_{i 8}\right)+\beta_{9} \ln \left(X_{i 9}\right)-U_{i}+V_{i}
\end{gathered}
$$

\footnotetext{
${ }^{19}$ Pour plus de détail, il faut consulter Baum, The Stata Journal (2008), 8, Numéro 2, pp 299303 et Wooldridge (2010) pp 748-755.

${ }^{20}$ Nous utilisons un signe négatif, afin de montrer que le terme $-U_{i}$ représente la différence entre la meilleure firme efficace (sur la frontière) et la firme observée.
} 
Avec $Y_{i}$ le rendement en maïs (en $\mathrm{kg} / \mathrm{ha}$ ) pour le producteur $i, X_{1}=\mathrm{la}$ quantité d'urée utilisée (en $\mathrm{kg} / \mathrm{ha}$ ), $X_{2}=$ la quantité de NPK utilisée (en $\mathrm{kg} / \mathrm{ha}$ ), $X_{3}=$ la quantité de semences utilisée (en $\mathrm{kg} / \mathrm{ha}$ ), $X_{4}=$ la quantité de main d'œuvre utilisée en homme-jour par hectare (en hj/ha), $X_{5}=$ la quantité d'herbicides utilisée (en L/ha), $X_{6}=$ la superficie exploitée (en ha), $X_{7}=$ montant du crédit formel obtenu (en FCFA), $X_{8}=$ montant du crédit informel obtenu (en FCFA) et $X_{9}=$ le climat (le niveau des précipitations).

Nous avons utilisé, dans le cadre de cette étude, des données secondaires comprenant principalement des données sur les producteurs de maïs, riz et cultures maraichères recueillies par le PAPA-INRAB ${ }^{21}$ en collaboration avec le Programme sous régional de la Gestion Intégrée de la Production et des Déprédateurs (GIPD) en 2012. Ces données fournissent des informations sur les caractéristiques, les pratiques de production, de récolte et post récolte des exploitations productrices du Maïs, riz et cultures maraichères dans six départements du Bénin à savoir : Collines, Couffo, Mono, Ouémé, Plateau et Zou. De façon précise, les données portent sur 203 producteurs et elles fournissent des informations économiques telles que la quantité en $\mathrm{kg}$ des inputs (engrais, urée, main d'œuvre, etc.) et de l'output, l'appartenance ou non à un groupement, le fait d'être en contact ou non avec un agent de vulgarisation, etc. Elles fournissent également des informations sur les caractéristiques du ménage telles les membres du ménage, le sexe, le statut d'alphabétisation et l'âge du chef de ménage, la distance qui sépare son habitation de son champ, etc. Le tableau 1 illustre la définition des variables qui seront utilisées dans nos modèles et le tableau 2 présente les caractéristiques principales de ces variables ainsi que la taille de l'échantillon.

\footnotetext{
${ }^{21}$ Institut National des Recherches Agricoles du Bénin
} 
Tableau 1 : Définition des variables qui sont utilisées dans nos modèles

Variable

Sexe de l'exploitant

Age de l'exploitant

Carré de l'âge

Climat

Alphabétisation

Taille du ménage

Montant du crédit formel

Montant du crédit informel

Superficie de l'exploitation

Prix du maïs

Appartenance à un

groupement de producteurs

Utilisation des semences améliorées

Contact avec un agent vulgarisateur

Contact avec une ONG

Alphabétisation $\times$ contact avec agent vulgarisateur

Alphabétisation $\times$ contact avec une ONG

Accès au champ

Zone cotonnière du Bénin

Zone des terres de barre

Zone de la dépression

Zone des pêcheries

Quantité produite

Herbicide

NPK

Urée

Semence

Main d'œuvre
Définition

1 si l'exploitant est de sexe masculin ; 0 sinon.

Age de l'exploitant en années

Carré de l'âge de l'exploitant

Mesuré par les précipitations

1 si l'exploitant est alphabétisé ; 0 sinon

Le nombre d'individus vivants dans le ménage

Montant du crédit formel obtenu pour la campagne agricole

Montant du crédit informel obtenu pour la campagne agricole

Superficie de l'exploitation en hectare (ha) Prix de vente du maïs

1 si l'exploitant est membre d'un groupement de producteurs ; 0 sinon

1 si l'exploitant utilise des semences améliorées ; 0 sinon

1 si l'exploitant utilise les services d'un agent vulgarisateur, 0 sinon

1 si l'exploitant est en contact avec une

ONG qui opère dans le domaine agricole, 0 sinon

1 si l'exploitant est alphabétisé et utilise les services de vulgarisation ; 0 sinon

1 si l'exploitant est alphabétisé et en contact avec une ONG, 0 sinon

1 si l'exploitant a accès à son champ en toute saison de l'année ; 0 sinon

1 si l'exploitant est dans la zone cotonnière du centre Bénin ; 0 sinon

1 si l'exploitant est dans la zone des terres de barre ; 0 sinon.

1 si l'exploitant est dans la zone de la dépression ; 0 sinon

1 si l'exploitant est dans la zone des pêcheries ; 0 sinon.

Quantité de maïs produite en $\mathrm{kg} / \mathrm{ha}$

Quantité d'herbicide utilisé en $\mathrm{L} / \mathrm{ha}$

Quantité de NPK utilisé en $\mathrm{kg} / \mathrm{ha}$

Quantité d'urée utilisée en $\mathrm{kg} / \mathrm{ha}$

Quantité de semences utilisées en $\mathrm{kg} / \mathrm{ha}$

Quantité de main d'œuvre utilisée en

homme/jour 
Tableau 2 : Statistiques descriptives des variables mobilisées pour le modèle d'efficacité technique

\begin{tabular}{|c|c|c|c|c|c|}
\hline $\mathrm{e}$ & Fréq & $(\%)$ & $\begin{array}{l}\text { Alphabétisation } \times \text { contact } \\
\text { avec une ONG }\end{array}$ & Fréq & $(\%)$ \\
\hline Masculin & 177 & 87,19 & Oui & 6 & 2,96 \\
\hline Féminin & 26 & 12,81 & Non & 197 & 97,04 \\
\hline $\begin{array}{l}\text { Appartenance } \\
\text { à un groupement }\end{array}$ & Fréq & $(\%)$ & $\begin{array}{l}\text { Utilisation de semences } \\
\text { améliorées }\end{array}$ & Fréq & $(\%)$ \\
\hline Oui & 20 & 9,85 & Oui & 30 & 14,78 \\
\hline Non & 183 & 90,15 & Non & 173 & 85,22 \\
\hline $\begin{array}{l}\text { Accès au } \\
\text { marché }\end{array}$ & Fréq & $(\%)$ & $\begin{array}{l}\text { Alphabétisation } \times \text { contact } \\
\text { avec agent vulgarisateur }\end{array}$ & Fréq & $(\%)$ \\
\hline Oui & 152 & 74,88 & Oui & 10 & 4,93 \\
\hline Non & 51 & 25,12 & Non & 193 & 95,07 \\
\hline Variable & & Moyenne & Ecart type & Minimum & Maximum \\
\hline Age de l'exploitant & & 45,0689 & 13,9676 & 17 & 89 \\
\hline Carré de l'âge & & 2225,34 & 1365,48 & 289 & 7921 \\
\hline climat & & 1112,04 & 107,922 & 963,7 & 1288,9 \\
\hline Taille du ménage & & 6,9655 & 3,8504 & 1 & 25 \\
\hline Superficie de & & 0,9249 & 1,0231 & 0,03 & 5,5 \\
\hline \multicolumn{6}{|l|}{ l'exploitation } \\
\hline Prix du maïs & & 173,743 & 29,5682 & 115 & 220 \\
\hline Quantité produite & & 1441,46 & 665,734 & 338 & 3250 \\
\hline Herbicide & & 53,8547 & 19,7716 & 1 & 65,5178 \\
\hline NPK & & 3,6287 & 2,1456 & 0,3333 & 18 \\
\hline Urée & & 18,1301 & 45,5417 & 2,7183 & 275 \\
\hline Semence & & 31,3876 & 32,8388 & 0,1667 & 137,5 \\
\hline Montant crédit forme & & 21280,7 & 112524 & 0 & 950000 \\
\hline \multirow{2}{*}{$\begin{array}{l}\text { Montant crédit } \\
\text { informel }\end{array}$} & & 2561,586 & 11228,49 & 0 & 80000 \\
\hline & & Nombre & bservations : 203 & & \\
\hline
\end{tabular}

\section{Résultats et discussions:}

Cette section présente successivement l'estimation des scores d'efficacité technique des producteurs et l'analyse des déterminants de leur niveau d'efficacité productive.

\section{Estimation du niveau d'efficacité technique des producteurs}

Les paramètres d'estimation de la fonction de production ont été obtenus en utilisant la fonction de type Cobb Douglas et la méthode MCO dans un premier temps puis la méthode de frontière stochastique dans un second temps. Nous reportons les effets marginaux moyens pour les deux modèles de la première étape. Le tableau 3 présente les résultats du modèle de la fonction de production par MCO et par SFA. Les résultats des MCO sont présentés dans la colonne marquée (1), tandis que ceux de la frontière stochastique sont montrés dans la colonne (2). 
En se concentrant sur le modèle de la fonction de production dans la colonne (2), nous observons que le coefficient de lambda est significatif à $1 \%$. Ce qui indique qu'il y a présence d'inefficacité technique chez les producteurs de l'échantillon. Le rendement potentiel n'est pas encore atteint. Cette valeur de lambda montre aussi que les producteurs pouvaient atteindre le rendement actuel avec moins de quantités d'intrants. Ce qui pose le problème de capacité des producteurs quant à la combinaison optimale des intrants de production.

Les résultats montrent que les intrants urée, NPK, semence, main d'œuvre, herbicide, superficie de l'exploitation ainsi que le climat ont un effet significatif sur la production des producteurs. En effet, le coefficient associé à l'urée est de 0.0805 , ce qui indique que l'effet moyen de l'urée sur le rendement est d'environ $8.05 \%$. Le coefficient associé à l'engrais NPK est de 0.1814 , ce qui indique que l'effet moyen des engrais NPK sur le rendement est d'environ $18.14 \%$. Ce résultat est proche de celui trouvé par Toléba et al. (2016). Le rendement est ainsi très positivement sensible aux variations de la quantité d'engrais NPK. La quantité de semence utilisée avec un coefficient de $7.03 \%$ a un effet positif et significatif sur le rendement du producteur. Il en est de même pour la main d'œuvre utilisée, les herbicides ainsi que le climat qui ont un effet moyen sur le rendement respectivement de 0.0087, 0.0617 et 0.6705 . Ce résultat montre l'importance des précipitations dans la production agricole et puisque Jalloh et al. (2013) prévoient une diminution ou une stabilité des précipitations au Sud du Bénin d'ici 2050 des stratégies d'adaptation doivent être envisagées.

La superficie de l'exploitation avec un coefficient de 5.45\% a un effet négatif et significatif sur le rendement. Ces résultats ne sont pas trop loin de ceux trouvés par Adegbola et al (2008) qui trouvent un effet moyen de 21\% pour l'engrais. Ces mêmes résultats ont été trouvés par Koirala et al. (2013) et Mohammed et Saghaian (2014).

Tableau $n^{\circ} 3$ : Estimations du modèle d'efficacité

\begin{tabular}{ccc}
\hline & \multicolumn{2}{c}{ Modèle de la fonction de production } \\
Variables & MCO $(1)$ & SFA $(2)$ \\
\hline Quantité urée & $0.1161^{* * *}$ & $0.0805^{* * *}$ \\
& $(0.030)$ & $(0.025)$ \\
Quantité npk & $0.2810^{* * *}$ & $0.1814^{* * *}$ \\
& $(0.082)$ & $(0.055)$ \\
Quantité semence & $0.0882^{* *}$ & $0.0703^{* * *}$ \\
& $(0.039)$ & $(0.024)$ \\
Quantité main d'œuvre & 0.0039 & $0.0087^{*}$ \\
& $(0.006)$ & $(0.005)$ \\
Quantité herbicide & $0.0987^{* * *}$ & $0.0617^{* *}$ \\
& $(0.037)$ & $(0.027)$ \\
Superficie & 0.0309 & $-0.0545^{*}$ \\
& $(0.031)$ & $(0.031)$ \\
Montant de crédit formel & 0.0065 & 0.0117 \\
& $(0.013)$ & $(0.008)$
\end{tabular}


Montant de crédit informel

Climat

Sigma2v

Sigma2u

Lambda

Sigma2

Constant

Observations

R-squared

LL
$0.0211 * *$

(0.010)

$1.1492 * * *$

(0.372)

(0.372)
0.0074

(0.010)

$0.6705^{* *}$

(0.297)

$-3.1809 * * *$

(0.379)

$-1.3412 * * *$

(0.223)

$2.5089 * * *$

(0.086)

$0.3031 * * *$

(0.050)

3.1505

(2.066)

203

Wald chi2(9)

$-0.8128$

(2.580)

203

0.162

\section{Distribution des scores d'efficacité des producteurs}

Les distributions de fréquence pour l'estimation des indices d'efficacité technique des producteurs sont présentées dans le tableau 4. Le score moyen d'efficacité technique des producteurs de notre échantillon est de $65,40 \%$, indiquant donc la présence d'inefficacité technique au niveau des producteurs. Le niveau d'efficacité technique varie entre $20,47 \%$ et $93,46 \%$. Nous supposons qu'un exploitant est dit efficace quand il obtient un indice supérieur ou égal au score moyen d'efficacité $(65,40 \%)$. Ainsi selon cette logique, seulement $61,08 \%$ des producteurs de notre échantillon sont techniquement efficaces.

Tableau $\mathrm{n}^{\circ} 4$ : Distribution des fréquences de l'efficacité technique des producteurs.

\begin{tabular}{|c|c|c|}
\hline \multirow[b]{2}{*}{ Efficacité (\%) } & \multicolumn{2}{|c|}{ Efficacité Technique } \\
\hline & Fréquence & Pourcentage $\%$ \\
\hline$\leq 25$ & 22 & 10,84 \\
\hline $26-49$ & 45 & 22,17 \\
\hline $50-60$ & 16 & 7,88 \\
\hline $61-70$ & 22 & 10,84 \\
\hline $71-80$ & 34 & 16,75 \\
\hline $81-90$ & 55 & 27,09 \\
\hline $91-100$ & 9 & 4,43 \\
\hline Moyenne (\%) & \multicolumn{2}{|c|}{65,40} \\
\hline Minimum (\%) & \multicolumn{2}{|c|}{20,47} \\
\hline Maximum (\%) & \multicolumn{2}{|c|}{93,46} \\
\hline
\end{tabular}




\section{Décomposition des scores d'efficacité selon les zones agro-écologiques}

Dans cette sous-section, nous présentons respectivement les niveaux d'efficacité des producteurs dans la zone cotonnière du centre Bénin ; la zone des terres de barre; la zone de la dépression et dans la zone des pêcheries. Cette décomposition des scores d'efficacité selon les zones agro-écologiques (tableau 5) révèle que les producteurs de la zone de la dépression sont plus techniquement efficaces comparativement aux producteurs des autres zones. Ceci montre que des efforts d'amélioration du niveau d'efficacité technique des producteurs doivent plus être orientés prioritairement vers les producteurs de la zone des pêcheries qui ont des niveaux d'efficacité technique les plus faibles, tout en ne négligeant pas ceux des autres zones.

Le score moyen d'efficacité technique des producteurs de la zone cotonnière du Bénin est de 65,34\% indiquant donc la présence d'inefficacité au niveau de ces producteurs. Dans cette zone, le niveau d'efficacité technique varie entre $22,16 \%$ et $93,46 \%$. Seulement $58,87 \%$ des producteurs de cette zone sont techniquement efficaces. Le score moyen d'efficacité technique des producteurs de la zone des terres de barre est de 74,23\%. Dans cette zone, le niveau d'efficacité varie entre $41,06 \%$ et $89,70 \%$ avec seulement $59,09 \%$ de producteurs techniquement efficaces. Celui des producteurs de la zone de la dépression est de 76,48\% avec un niveau d'efficacité technique variant entre $50,28 \%$ et $91,51 \%$. Environ $53,85 \%$ des producteurs de cette zone sont techniquement efficaces. Enfin, les producteurs de la zone de pêcheries ont un score moyen de 50,07\%. Dans cette zone, le niveau d'efficacité technique varie entre $20,47 \%$ et $90,95 \%$ avec seulement $41,94 \%$ de producteurs techniquement efficaces.

Tableau n ${ }^{\circ} 5$ : Fréquences des efficacités techniques des producteurs des différentes zones agro écologiques du Bénin

\begin{tabular}{|c|c|c|c|c|c|c|c|c|}
\hline \multirow[b]{3}{*}{$\begin{array}{c}\text { Efficacité } \\
(\%)\end{array}$} & \multirow{2}{*}{\multicolumn{2}{|c|}{\begin{tabular}{|c|} 
Zone \\
cotonnière du \\
Bénin
\end{tabular}}} & \multirow{2}{*}{\multicolumn{2}{|c|}{$\begin{array}{c}\begin{array}{c}\text { Zone des } \\
\text { terres de barre }\end{array} \\
\text { Technique }\end{array}$}} & \multicolumn{2}{|c|}{$\begin{array}{l}\text { Zone de la } \\
\text { dépression }\end{array}$} & \multicolumn{2}{|c|}{$\begin{array}{l}\text { Zone des } \\
\text { pêcheries }\end{array}$} \\
\hline & & & & & \multicolumn{2}{|c|}{ Technique } & \multicolumn{2}{|c|}{ Technique } \\
\hline & Fréq & $\%$ & Fréq & $\%$ & Fréq & $\%$ & Fréq & $\%$ \\
\hline$<\mathbf{5 0}$ & 39 & 31,45 & 5 & 22,73 & 3 & 11,54 & 20 & 64,52 \\
\hline $50-100$ & 85 & 68,55 & 17 & 77,27 & 23 & 88,46 & 11 & 35,48 \\
\hline $\operatorname{Moy}(\%)$ & & 34 & & 23 & & 76,48 & & 50,07 \\
\hline $\operatorname{Min}(\%)$ & & 16 & & 06 & & 50,28 & & 20,47 \\
\hline $\operatorname{Max}(\%)$ & & 46 & & 70 & & 91,51 & & 90,95 \\
\hline
\end{tabular}

\section{Décomposition des scores d'efficacité selon le type de semences utilisées}

Une analyse attentive du tableau 6 montre que les producteurs qui utilisent des semences améliorées ont un score moyen d'efficacité technique statistiquement plus élevé que celui des producteurs qui utilisent des semences traditionnelles. Ce qui montre que les semences améliorées doivent être 
rendues plus accessibles aux producteurs et surtout à moindre coût afin d'améliorer leur niveau d'efficacité. Parmi les producteurs qui utilisent les semences améliorées, $86,67 \%$ sont techniquement efficaces contre $63,58 \%$, pour ceux qui utilisent des semences traditionnelles. Ce qui ne fait que confirmer l'importance des semences améliorées dans l'amélioration du rendement des producteurs.

Tableau nº6 : Décomposition des scores d'efficacité selon les types de semences utilisées Utilisation des semences traditionnelles Utilisation des semences ttest khi 2 Technique améliorées

\begin{tabular}{|c|c|c|c|c|c|}
\hline \multirow[b]{2}{*}{ Efficacité (\%) } & \multicolumn{2}{|c|}{ Technique } & \multicolumn{2}{|c|}{ Technique } & \\
\hline & Fréquence & $\%$ & Fréquence & $\%$ & \\
\hline$<\mathbf{5 0}$ & 63 & 36,42 & 4 & 13,33 & \\
\hline $50-100$ & 110 & 63,58 & 26 & 86,67 & \\
\hline Moyenne (\%) & & & & & $-11,21 * * *$ \\
\hline $\begin{array}{c}\text { Minimum } \\
(\%)\end{array}$ & & & & & \\
\hline Maximum(\%) & & & & & \\
\hline
\end{tabular}

Facteurs affectant les niveaux d'efficacité techniques des producteurs

Pour identifier les facteurs qui déterminent les niveaux d'efficacité des producteurs, nous utilisons dans un premier temps le Fractional Regression Model (FRM) et afin d'analyser la robustesse de ces facteurs identifiés, nous utilisons dans un second temps le modèle GLM puis les MCO. Les résultats de ces différentes estimations sont consignés dans le tableau 7. Les signes négatifs des paramètres du tableau 7 signifient que les variables associées ont un effet positif sur l'efficacité technique du producteur puisque la variable dépendante est le score d'inefficacité technique.

Selon les résultats, un exploitant de sexe masculin a une faible probabilité d'être techniquement inefficace comparativement à une exploitante de sexe féminin. Ce résultat pourrait être expliqué par les facteurs liés au cycle de vie du ménage. Les ménages dirigés par les femmes ont tendance à avoir plus de membres que ceux dirigés par les hommes ( 8 contre 6$)$. Par conséquent, les femmes chefs de ménage peuvent passer moins de temps dans les activités de production rémunératrice. Une autre interprétation est que les hommes chefs de ménage ont des compétences managériales supérieures, ont moins de contrainte de main d'œuvre dans les activités de production agricole. Les femmes sont généralement moins capables que les hommes de garantir les droits à la terre, ou de garantir un plus grand accès à la terre. Les rigidités des droits à la terre et à la main d'œuvre dans le ménage ou la communauté ensemble avec un fort contrôle typiquement exercé par les hommes contribuent à une faible efficacité technique.

De plus, les exploitants qui utilisent des semences améliorées ont une probabilité forte d'être techniquement efficaces comparativement à ceux qui utilisent les semences traditionnelles. Ce résultat peut s'expliquer par le fait 
que l'utilisation des semences améliorées se traduit par une amélioration du niveau de production et donc de l'efficacité technique. Ce résultat est conforme à celui trouvé par Toléba et al. (2016) et Adegbola et al. (2005).

Plus le prix de vente du maïs est élevé, la probabilité que le producteur soit techniquement efficace augmente. Ce qui montre le rôle important que jouent les politiques de maintien des prix de vente de cette céréale dans une fourchette plus ou moins stable. Plus le prix est relativement stable, plus le producteur est en confiance et il s'implique davantage pour améliorer son rendement.

Les revenus non agricoles ont un effet négatif et significatif sur l'inefficacité technique des producteurs. Si les marchés des capitaux fonctionnaient comme cela se doit, l'introduction d'autres sources de revenus ne devrait pas affecter l'efficacité technique (Chavas et al. 2005). Nos résultats indiquent une présence d'un mauvais fonctionnement des marchés des capitaux/crédits où les contraintes de liquidités sont surmontées à travers des revenus issus des activités non agricoles.

Les résultats montrent également que les exploitants qui sont à la fois alphabétisés et en contact avec une ONG opérant dans le secteur agricole ont une forte probabilité d'être techniquement efficaces comparativement à ceux qui ne sont pas. Ceci s'explique par le fait que l'éducation peut accroitre l'acquisition d'informations et l'habileté d'ajustement des producteurs et donc leur capacité de mise en application des informations et formations données par l'ONG dans le cadre de leur activité. En plus, cela pourra les aider à adopter des technologies agricoles modernes pouvant leur permettre d'atteindre un niveau de production plus élevé avec les mêmes niveaux d'intrants. Ce résultat est conforme à ceux obtenus par Ogundari (2013); Costa et al (2013) ; de Magalhães et al. (2011) ; Asefa (2011) ; Lius et Myers (2009).

Un exploitant alphabétisé et en contact avec un agent vulgarisateur a une forte probabilité d'être techniquement inefficace comparativement à ceux qui ne le sont pas, les autres facteurs constants. Ce résultat est contraire à celui trouvé par Toléba et al. (2016) mais ce résultat pourrait s'expliquer par le fait que dans les années 60 les services de vulgarisation fut assurés par l'Etat. Après 1990, l'Etat a décidé du transfert de l'encadrement aux producteurs ainsi chaque producteur devrait supporter les coûts liés aux services des agents vulgarisateurs à chaque fois qu'il en a besoin. Cet état de chose a fait que les services de vulgarisation ont peu à peu disparu dans le processus de production puisque les producteurs n'ont pas les moyens de s'assurer ces services de vulgarisation.

A l'avènement d'un nouveau régime en 2006, les nouvelles autorités du pays ont décidé de renouer avec les services de vulgarisation. Cette volonté politique les a amenées à recruter de jeunes agents de vulgarisation qui viennent de finir leur formation sans aucune expérience. Ce qui a engendré 
une crise de confiance entre ces agents de vulgarisation et les producteurs qui s'estiment être plus expérimentés et plus outillés que ces derniers. Cette crise de confiance amène les producteurs à ne pas suivre à la lettre les instructions de ces agents vulgarisateurs. Ce résultat pourrait aussi s'expliquer par une insuffisance dans l'intensité des conseils de ces agents ou par un manque de régularité. Mais aussi, les producteurs peuvent prendre une instruction donnée par rapport à une situation particulière comme applicable à toute situation donnée.

Une autre explication possible est que les agents vulgarisateurs ne tiennent pas compte des réalités endogènes et des spécificités liées à chaque zone d'intervention pour conseiller les producteurs. Ce qui pose le problème de contexte et/ou de la qualité des services fournis par les services de vulgarisation. Une stratégie visant à améliorer les services de vulgarisation semble désormais être nécessaire afin d'atteindre les résultats escomptés.

L'accès au crédit informel a un effet négatif et significatif sur le niveau d'inefficacité technique des producteurs. Il en est de même pour l'accès au crédit formel mais l'effet n'est pas significatif. Ce qui indique les producteurs qui ont accès au crédit ont une forte probabilité d'être techniquement efficaces comparativement aux autres producteurs. Ce résultat s'explique par le fait que la disponibilité du crédit (surtout informel qui est plus accessible aux producteurs agricoles au Bénin) saute la contrainte de liquidité et permet aux producteurs de faire des achats d'intrants en temps opportun qu'ils ne peuvent pas se permettre autrement à partir de leurs propres ressources et améliore l'utilisation des intrants agricoles qui mène à une plus grande efficacité. Ce résultat est conforme à celui trouvé par Toléba et al. (2016) et Asefa (2011).

Les producteurs de la zone des pêcheries ont une forte probabilité d'être techniquement inefficaces comparativement aux producteurs des autres zones. Ce résultat pourrait être expliqué par la qualité des sols qu'on retrouve dans chaque zone agro écologique. Ce qui montre que les politiques d'amélioration de l'efficacité des producteurs doivent prendre en compte la spécificité de chaque zone pour avoir plus d'impact en définissant les qualités et type d'intrants qui correspondent à chaque zone agro écologique. 
Tableau $n^{\circ}$ 7: Facteurs affectant les niveaux d'inefficacité technique des producteurs

\begin{tabular}{|c|c|c|c|}
\hline VARIABLES & $\begin{array}{c}(1) \\
\text { FRM } \\
\end{array}$ & $\begin{array}{c}(2) \\
\text { GLM } \\
\end{array}$ & $\begin{array}{c}(3) \\
\mathrm{MCO} \\
\end{array}$ \\
\hline Sexe de l'exploitant (masculin $=1$ ) & $\begin{array}{c}-0.3897 * * \\
(0.158)\end{array}$ & $\begin{array}{c}-0.0863 * * * \\
(0.033)\end{array}$ & $\begin{array}{c}-0.0863^{* *} \\
(0.035)\end{array}$ \\
\hline Age de l'exploitant & 0.0332 & 0.0070 & 0.0070 \\
\hline Age au carré & $\begin{array}{r}-0.0005 \\
(0.000)\end{array}$ & $\begin{array}{c}-0.0001 \\
(0.000)\end{array}$ & $\begin{array}{r}-0.0001 \\
(0.000)\end{array}$ \\
\hline Taille du ménage & $\begin{array}{l}0.0072 \\
(0.016)\end{array}$ & $\begin{array}{l}0.0017 \\
(0.004)\end{array}$ & $\begin{array}{l}0.0017 \\
(0.004)\end{array}$ \\
\hline Appartenance à un groupement (oui $=1$ ) & $\begin{array}{l}0.0889 \\
(0.187)\end{array}$ & $\begin{array}{l}0.0198 \\
(0.043)\end{array}$ & $\begin{array}{l}0.0198 \\
(0.045)\end{array}$ \\
\hline Utilisation des semences améliorées (oui $=1$ ) & $\begin{array}{c}-0.614 * * * \\
(0.186)\end{array}$ & $\begin{array}{c}-0.1263 * * * \\
(0.036)\end{array}$ & $\begin{array}{c}-0.1263 * * * \\
(0.038)\end{array}$ \\
\hline Prix de vente du maïs & $\begin{array}{c}-0.007 * * * \\
(0.002)\end{array}$ & $\begin{array}{c}-0.0015^{* * * *} \\
(0.000)\end{array}$ & $\begin{array}{c}-0.0015^{* * * *} \\
(0.001)\end{array}$ \\
\hline Accès au champ & $\begin{array}{l}0.3930 \\
(0.254)\end{array}$ & $\begin{array}{l}0.0855 \\
(0.053)\end{array}$ & $\begin{array}{l}0.0855 \\
(0.055)\end{array}$ \\
\hline Part du revenu non agricole & $\begin{array}{c}-0.0530 * \\
(0.032)\end{array}$ & $\begin{array}{c}-0.0106^{*} \\
(0.006)\end{array}$ & $\begin{array}{c}-0.0106^{*} \\
(0.006)\end{array}$ \\
\hline Alphabétisation $\times$ contact avec agent vulgarisateur & $\begin{array}{c}0.7021 * * * \\
(0.264)\end{array}$ & $\begin{array}{c}0.1430 * * \\
(0.059)\end{array}$ & $\begin{array}{c}0.1430 * * \\
(0.062)\end{array}$ \\
\hline Alphabétisation $\times$ contact avec une $\mathrm{ONG}$ & $\begin{array}{c}-1.003 * * * \\
(0.240)\end{array}$ & $\begin{array}{c}-0.1951 * * * \\
(0.048)\end{array}$ & $\begin{array}{c}-0.1951 * * * \\
(0.050)\end{array}$ \\
\hline Accès au crédit informel (oui $=1)$ & $\begin{array}{c}-0.3828^{*} \\
(0.208)\end{array}$ & $\begin{array}{c}-0.0919^{*} \\
(0.050)\end{array}$ & $\begin{array}{c}-0.0919^{*} \\
(0.052)\end{array}$ \\
\hline Accès au crédit formel (oui $=1)$ & $\begin{array}{l}-0.2388 \\
(0.227)\end{array}$ & $\begin{array}{l}-0.0526 \\
(0.053)\end{array}$ & $\begin{array}{l}-0.0526 \\
(0.056)\end{array}$ \\
\hline Zone cotonnière du Bénin & $\begin{array}{c}-0.8415^{* *} \\
(0.349)\end{array}$ & $\begin{array}{c}-0.2004 * * * \\
(0.076)\end{array}$ & $\begin{array}{c}-0.2004 * * \\
(0.079)\end{array}$ \\
\hline Zone des terres de barre & $\begin{array}{c}-1.407 * * * \\
(0.293)\end{array}$ & $\begin{array}{c}-0.3176^{* * *} \\
(0.064)\end{array}$ & $\begin{array}{c}-0.3176^{* * * *} \\
(0.066)\end{array}$ \\
\hline Zone de la dépression & $\begin{array}{c}-1.567 * * * \\
(0.386)\end{array}$ & $\begin{array}{c}-0.3464 * * * \\
(0.084)\end{array}$ & $\begin{array}{c}-0.3464 * * * \\
(0.087)\end{array}$ \\
\hline Constant & $\begin{array}{c}-1.8744 * * \\
(0.775)\end{array}$ & $\begin{array}{l}0.1114 \\
(0.168)\end{array}$ & $\begin{array}{l}0.1114 \\
(0.175)\end{array}$ \\
\hline $\begin{array}{c}\text { Observations } \\
\text { R-squared }\end{array}$ & 203 & 203 & $\begin{array}{c}203 \\
0.270\end{array}$ \\
\hline
\end{tabular}

Ecart-types robustes entre parenthèses

$* * * \mathrm{p}<0.01, * * \mathrm{p}<0.05, * \mathrm{p}<0.1$

\section{Conclusion et implications politiques:}

Cette étude utilise la frontière de production pour estimer le niveau d'efficacité technique des producteurs en utilisant les données d'inputs et d'output de 203 producteurs du maïs issus de six départements du Bénin. Les résultats montrent que le niveau moyen d'efficacité technique est de $65,40 \%$. Parmi ces producteurs de l'échantillon, 61,08\% sont techniquement efficaces. 
Une décomposition des scores d'efficacité selon les zones agro-écologiques révèle que les producteurs de la zone de dépression sont plus efficaces techniquement que ceux des autres zones. Les résultats indiquent également que les producteurs qui utilisent des semences améliorées sont statistiquement plus efficaces techniquement que les autres.

L'analyse de la seconde étape révèle que les variables telles que le sexe de l'exploitant, l'utilisation des semences améliorées, le prix de vente du maïs, la part du revenu non agricole, le contact avec une ONG, l'accès au crédit (informel) ainsi que la zone de production ont un rôle positif dans l'atteinte de la frontière de production.

En termes d'implications politiques, nos résultats montrent que la politique de distribution des semences améliorées et des engrais du gouvernement aide les producteurs à être plus efficaces contrairement à celle d'utilisation des agents de vulgarisation. Ce résultat nous amène à suggérer que le gouvernement peut économiser les ressources utilisées dans le cadre des services de vulgarisation et se focaliser sur sa politique de distribution des semences améliorées en investissant plus dans la recherche des semences améliorées. Ce qui suppose une mise à disposition des ressources suffisantes aux centres de recherche spécialisés dans la production des semences améliorées afin de permettre aux producteurs de les avoir à un coût raisonnable.

Une politique d'amélioration des services de vulgarisation s'avère indispensable et celle-ci peut consister par exemple à avoir avec les agents vulgarisateurs un contrat salarial ayant deux composantes: une composante fixe et une autre qui est fonction du niveau de productivité des producteurs encadrés par ces derniers. Ainsi, la recherche permanente d'un salaire élevé va les amener à mieux se donner dans l'encadrement et le suivi des producteurs. Une sensibilisation des producteurs est aussi nécessaire pour créer une ambiance de confiance entre les agents vulgarisateurs et les producteurs.

La création des incitations au niveau institutionnel pour accroitre l'offre de crédits afin de mettre en place un fonds de crédits agricoles permettant aux producteurs d'avoir accès à des crédits agricoles à taux préférentiels et au temps opportun est nécessaire pour corriger les imperfections sur le marché du capital et/ou du travail.

D'autres actions permettant une amélioration du niveau d'efficacité des producteurs doivent être poursuivies. Par exemple, l'investissement dans l'éducation et l'alphabétisation des producteurs. Des politiques de stabilisation des prix de vente du maïs et d'aménagement des pistes rurales doivent aussi être poursuivies pour garantir aux producteurs une certaine assurance dans l'écoulement de leur production. Les politiques d'adaptation aux effets du 
changement climatique doivent être renforcées à travers par exemple la pratique de l'irrigation.

\section{Remerciements :}

Nous voudrions adresser nos remerciements au Consortium pour la Recherche Economique en Afrique (CREA) pour avoir accepté financer cette recherche. Nos remerciements vont également à l'endroit des personnes ressources ainsi que les chercheurs participants aux différents biannuels pour leurs importants commentaires et suggestions.

\section{Références:}

1. Abdulai, A., et Tietje, H. (2007). Estimating technical efficiency under unobserved heterogeneity with stochastic frontier models: application to northern German dairy farms. European Review of Agricultural Economics, 34(3), 393-416. doi:10.1093/erae/jbm023

2. Adegbola, P., Sossou, H., Singbo, A., et Sodjinou, E. (2008). Analyse de l'efficacité technique et économique dans les systèmes rizicoles du Centre et du Nord-Est du Bénin. Vasa (pp. 1-17).

3. Ahmad, M. (2003). Agricultural Productivity, Efficiency, and Rural Poverty in Irrigated Pakistan: A Stochastic Production Frontier Analysis. The Pakistan Development Review, 42(3), 219-248.

4. Aigner, D. J., Lovell, C. A. K., et Schmidt, P. (1977). Formulation and estimation of stochastic frontier production function models. Journal of Econometrics, 6, 21-37.

5. Alene, A. D., et Hassan, R. M. (2006). The Efficiency of Traditional and Hybrid Maize Production in Eastern Ethiopia: An Extended Efficiency Decomposition Approach. Journal of African Economies, 15(2), i-Xxvii. doi:10.1093/jae/ej1020

6. Banker, R. D., Charnes, A., et Cooper, W. W. (1984). Some models for estimating technical and scale inefficiencies in data envelopment analysis. Management Science, 30(9), 1078-1092.

7. Battese, G., et Coelli, T. (1995). A model for technical inefficiency effects in a stochastic frontier production function for panel data. Empirical Economics, 325-332.

8. Battese, G.E., S. J. Malik et M.A. Gill (1996) 'An investigation of technical inefficiencies of production of wheat farmers in fours districts of Pakistan', Journal of Agricultural Economics, (47): 37-49.

9. Baum, C. (2008). Stata tip 63: Modeling proportions. Stata Journal, (2), 299-303. Retrieved from http://ageconsearch.umn.edu/bitstream/122595/2/sjart_st0147.pdf 
10. Chen, Z., Huffman, W. E., et Rozelle, S. (2009). Farm technology and technical efficiency: Evidence from four regions in China. China Economic Review, 20(2), 153-161. doi:10.1016/j.chieco.2009.03.002

11. Charnes, A., Cooper, W. W., Rhodes, E. (1978). Measuring the efficiency of decision making units. European Journal of Operational Research, 2(6), 429-444.

12. Chavas, J.-P., Petrie, R., et Roth, M. (2005). Farm household production efficiency : Evidence from the Gambia. American Journal of Agricultural Economics, 87(February), 160-179.

13. Christensen, L. R., D. W. Jorgenson, L. J. Lau (1971) "Conjugate duality and the transcendental logarithmic function". Econometrica, 39 (4).

14. Costa, L. V., Gomes, M. F. M., et de Lelis, D. A. S. (2013). Food Security and Agricultural Productivity in Brazilian Metropolitan Regions. Procedia Economics and Finance, 5(13), 202-211. doi:10.1016/S2212-5671(13)00026-9

15. Debreu, D. G. (1951) "The Coefficient of Resource Utilisation." Econometrica, 19: 273-292.

16. De Magalhães, M. M., Filho, H. M. de S., de Sousa, M. R., da Silveira, J. M. F. J., et Buainain, A. M. (2011). Land Reform in NE Brazil : a stochastic frontier production. RESR, Piracicaba, 49(01), 9-30.

17. Dhungana, B. R., Nuthall, P. L., et Nartea, G. V. (2004). Measuring the economic inefficiency of Nepalese rice farms using data envelopment analysis. The Australian Journal of Agricultural and Resource Economics, 48(2), 347-369. doi:10.1111/j.14678489.2004.00243.x

18. Dinar, A., Karagiannis, G., et Tzouvelekas, V. (2007). Evaluating the impact of agricultural extension on farms' performance in Crete: a nonneutral stochastic frontier approach. Agricultural Economics, 36(2), 135-146. doi:10.1111/j.1574-0862.2007.00193.x

19. Djimasra, N. (2010). Mesure de l'efficacité technique des pays africains producteurs du coton : une application de la méthode Data Envelopment Analysis (DEA) (No. 2010-28) (Vol. 33, pp. 0-36).

20. ERKOC, TE. 2012. "Estimation Methodology of Economic Efficiency: Stochastic Frontier Analysis vs Data Envelopment Analysis." International Journal of Academic Research in Economics and Management Sciences 1(1):1-23.

21. Farrell, M. J. (1957). The measurement of productive efficiency. Journal of the Royal Statistical Society, 120(3), 253-290.

22. Fiorentino, E., Karmann, A., et Koetter, M. (2006). The cost efficiency of German banks : a comparison of SFA and DEA (No. 10). 
23. Huang, C.J. et J.T. Liu (1994) "Estimation of a Non-Neutral Stochastic Frontier Production Function," Journal of Productivity Analysis, 5: 171-180.

24. Idiong, I. C. (2007). Estimation of Farm Level Technical Efficiency in Smallscale Swamp Rice Production in Cross River State of Nigeria : A Stochastic Frontier Approach. World Journal of Agricultural Sciences, 3(5), 653-658.

25. Jalloh, A., Nelson, G. C., Thomas, T. S., Zougmoré, R. B., \& RoyMacauley, H. (Eds.). (2013). West African Agriculture and Climate Change: A Comprehensive Analysis. International Food Policy Research Institute. Washingston, DC, USA.

26. Kalirajan, K. (1991) "The importance of efficient use in the adoption of technology: A micro panel data analysis. " Journal of Productivity Analysis, 2: 113-126.

27. Koirala, K. H., Mishra, A. K., et Mohanty, S. (2013). Determinants of Rice Productivity and Technical Efficiency in the Philippines. In Southern Agricultural Economics Association (SAEA) Annual Meeting (pp. 1-16).

28. Koopmans, T. C. (1951) An analysis of production as an efficient combination of activities, dans Activity Analysis of production and allocation, ed. par T.C. Koopmans, Cowles Commission for research in Economics, Monograph 13. New York: John-Wiley and Sons, Inc.

29. Kumbhakar, S. C., Ghosh, S., et McGuckin, J. T. (1991). A Generalized Production Frontier Approach for Estimating Determinants of Inefficiency in U.S. Dairy Farms. Journal of Business et Economic Statistics, 9(3), 279-286.

30. Kumbhakar, S. C., et Lovell, C. A. K. (2000). Stochastic frontier analysis. Economica (Vol. 69, p. 680). Cambridge University press.

31. Liu, Y. et R. Myers (2009) "Model selection in stochastic frontier analysis with an application to maize production in Kenya." Journal of Productivity Analysis, 31: 3346.

32. Leibenstein, H. (1966). Allocative Efficiency vs. "X-Efficiency." The American Economic Review, 56(3), 392 - 415.

33. Loureiro, M. L. (2009). Farmers' health and agricultural productivity. Agricultural Economics, 40(4), 381-388. doi:10.1111/j.15740862.2009.00385.X

34. Meeusen, W., et Broeck, J. Van Den. (1977). Efficiency estimation from Cobb-Douglas production functions with composed error. International Economic Review, 18(2), 435-444.

35. Mohammed, R., et Saghaian, S. (2014). Technical Efficiency Estimation of Rice Production in South Korea. In Southern 
Agricultural Economics Association (SAEA) Annual Meeting (pp. 116).

36. Nasim, S., Dinar, A., et Helfand, S. (2014). Allocative Inefficiency and Farm-Level Constraints in Irri-gated Agriculture in Pakistan. Ifpri.org, (015), 1-67.

37. Ogundari, K. (2013). The Paradigm of African Agricultural Efficiency, 1967-2012: What Does Meta-Analysis Reveal? Conference, August 28-30, 2013, Christchurch, (24), 1-27.

38. Otieno, DJ, Lionel Hubbard, et Eric Ruto. 2012. "Determinants of Technical Efficiency in Beef Cattle Production in Kenya." International Association of Agricultural Economists (IAAE) Triennial Conference, Foz do Iguacu, Brasil, 18-24 August, 2012. 18-24.

39. Papke, L. E., et Wooldridge, J. M. (1996). Econometric methods for fractional response variables with an application to $401(\mathrm{k})$ plan participation rates, 11(February), 619-632.

40. Ramalho, E. A., Ramalho, J. J. S., et Murteira, J. M. R. (2009). Alternative estimating and testing empirical strategies for fractional regression models (No. 08) (pp. 1-66).

41. Ramalho, E., Ramalho, J., et Henriques, P. (2010). Fractional regression models for second stage DEA efficiency analyses (No. 01). ... of Productivity Analysis (Vol. 34, pp. 239-255).

42. Ray, S. (1988) "Data envelopment analysis, nondiscretionary inputs and efficiency: an alternative interpretation." Socio-Economics. Plann. Sci. 22, p167-176.

43. Singh, I., Squire, L. et Stauss, J. (1986). Agricultural Household Models. Extensions, Applications, and Policy. The World Bank. John Hopkins University Press, Baltimore and London.

44. Thirtle, C., Lin, L., \& Piesse, J. (2003). The impact of research-led agricultural productivity growth on poverty reduction in Africa, Asia and Latin America. World Development.

45. Toléba, S. M., Biaou, G., Zannou, A., \& Saïdou, A. (2016). Évaluation Du Niveau D’efficacité Technique Des Systèmes De Production A Base De Maïs Au Bénin. European Scientific Journal, 12(27), 276299. http://doi.org/10.19044/esj.2016.v12n27p276

46. Venkataramani, a, Shanmugam, K., et Ruger, J. (2010). Health, Technical Efficiency And Agricultural Production In Indian Districts. Journal of Economic Development, 35(4), 1-23.

47. Wooldridge, J. M. 2010. Econometric Analysis of Cross Section and Panel Data. Second Edition, The MIT Press, Cambridge, Massachusetts, London, England. 